TITLE:

\title{
Ultrashort-pulse laser calligraphy
}

$\operatorname{AUTHOR}(\mathrm{S})$ :

Yang, Weijia; Kazansky, Peter G.; Shimotsuma, Yasuhiko; Sakakura, Masaaki; Miura, Kiyotaka; Hirao, Kazuyuki

\section{CITATION:}

Yang, Weijia ... [et al]. Ultrashort-pulse laser calligraphy. APPLIED PHYSICS LETTERS 2008, 93(17): 171109.

\section{ISSUE DATE:}

2008-10-27

URL:

http://hdl.handle.net/2433/84591

\section{RIGHT:}

Copyright 2008 American Institute of Physics. This article may be downloaded for personal use only. Any other use requires prior permission of the author and the American Institute of Physics. 


\title{
Ultrashort-pulse laser calligraphy
}

\author{
Weijia Yang, ${ }^{1}$ Peter G. Kazansky, ${ }^{1, a)}$ Yasuhiko Shimotsuma, ${ }^{2}$ Masaaki Sakakura, ${ }^{2}$ \\ Kiyotaka Miura, ${ }^{2}$ and Kazuyuki Hirao ${ }^{2}$ \\ ${ }^{1}$ Optoelectronics Research Centre, University of Southampton, SO17 1BJ, United Kingdom \\ ${ }^{2}$ Department of Material Chemistry, Graduate School of Engineering, Kyoto University, Katsura, \\ Nishikyo-ku, Kyoto 615-8510, Japan
}

(Received 19 September 2008; accepted 9 October 2008; published online 29 October 2008)

\begin{abstract}
Control of structural modifications inside silica glass by changing the front tilt of an ultrashort pulse is demonstrated, achieving a calligraphic style of laser writing. The phenomena of anisotropic bubble formation at the boundary of an irradiated region and modification transition from microscopic bubbles formation to self-assembled form birefringence are observed, and the physical mechanisms are discussed. The results provide the comprehensive evidence that the light beam with centrosymmetric intensity distribution can produce noncentrosymmetric material modifications. (C) 2008 American Institute of Physics. [DOI: 10.1063/1.3010375]
\end{abstract}

Modification of transparent materials with ultrafast lasers has attracted considerable interest due to a wide range of applications including laser surgery, ${ }^{1}$ integrated optics, ${ }^{2}$ optical data storage, ${ }^{3}$ and three-dimensional micro- $^{4,5}$ and nanostructuring. ${ }^{6,7}$ Three different types of structural changes can be induced with ultrafast laser irradiation in the bulk of a transparent material, silica glass in particular: an isotropic refractive index change (type 1 ), ${ }^{2}$ a form birefringence associated with self-assembled nanogratings and negative refractive index change (type 2 ), ${ }^{7}$ and a void (type 3 ). ${ }^{3}$ The type of modification depends on several factors including the laser parameters (wavelength, pulse duration, energy, and repetition rate), the numerical aperture (NA) of a focusing lens and the material parameters (band gap, thermal properties). In fused silica, the transition from type 1 to type 2 and finally to type 3 modification is observed with an increase of pulse energy. More recently, a remarkable phenomenon in ultrafast laser processing of transparent materials has been reported manifesting itself as a change in material modification by reversing the writing direction. ${ }^{8}$ It has been speculated that the pulse front tilt could be added to the list of laser parameters defining the light-matter interactions in material processing. One of the main reasons why this peculiar physical phenomenon has not been observed until recently is explained by the difficulty in the measurement and control of the tilt of intensity front of an ultrashort pulse. Sophisticated nonlinear optical techniques such as frequency resolved optical gating and its modifications (GRENOUILLE) have been developed for characterization of the pulse front tilt. ${ }^{9}$

In this letter, we provide experimental evidence, which shows that indeed the pulse front tilt can be used to control material modifications and in particular as a tool for laser processing and optical manipulation, e.g., for achieving calligraphic style of laser writing, when the appearance of a "stroke" varies in relation to its direction. Moreover, anisotropic bubble formation and bubble-nanograting transition were observed at high pulse energies. Our experiments using pulse front tilt characterization and control provide the experimental evidence that the tilt in intensity front of an ul-

${ }^{\text {a)} E l e c t r o n i c ~ m a i l: ~ p g k @ o r c . s o t o n . a c . u k . ~}$ trashort pulse can reveal itself directly in laser induced material modifications.

An amplified, mode-locked Ti:sapphire laser operating at $800 \mathrm{~nm}$ wavelength with $70 \mathrm{fs}$ pulse duration and a $250 \mathrm{kHz}$ repetition rate was used in the first experiment. The linearly polarized laser beam was focused via a $50 \times(\mathrm{NA}=0.8) \mathrm{ob}-$ jective at a depth of $60 \mu \mathrm{m}$ beneath the surface of the fused silica sample. Line structures were written inside the bulk material by translating the sample perpendicularly to the light propagation direction, using a linear motorized stage (Aerotech ALS-130). After irradiation, the sample was inspected using an optical microscope. The first group of lines was written by scanning in alternating directions inside the sample with the pulse energy of $2.6 \mu \mathrm{J}$ and the scan speed of $50 \mu \mathrm{m} / \mathrm{s}$ [Fig. 1(a)]. The temporal characteristic of the pulses, in particular the pulse front tilt before the focusing objective, were characterized using a GRENOUILLE device (8.50 Model). The measured pulse front tilt for the first group of lines was $4 \times 10^{-2} \mathrm{fs} / \mathrm{mm}$. As shown in [Fig. 1(a)], the directional dependence can be clearly observed in the morphology of the lines written in opposite directions, with a line written in one direction being rougher than a line written in the reversed direction. The directional dependence can also be revealed by imaging the lines between crossed polarizers, in which only the smooth lines written in one direction show birefringence. Next the pulse front tilt was reversed

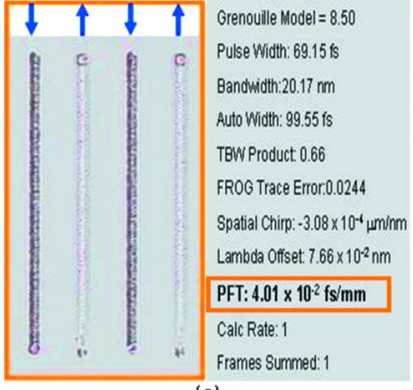

(a)

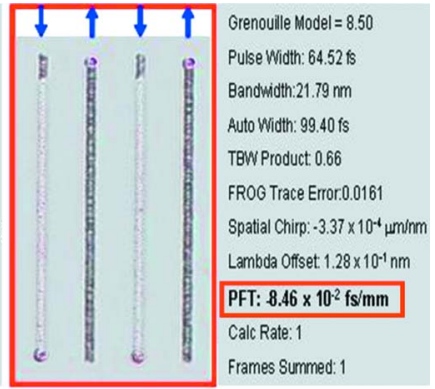

(b)
FIG. 1. (Color online) Microscope bright field image of the line structures written using femtosecond pulses with (a) positive pulse front tilt or (b) negative pulse front tilt. The distance between the lines is $25 \mu \mathrm{m}$. The writing direction is shown by the arrow. The respective screen shots containing measured laser pulse parameters by GRENOUILLE device are shown. 
by tuning the pulse compressor to the value of -8.64 $\times 10^{-2} \mathrm{fs} / \mathrm{mm}$ and the second group of lines was imprinted by scanning in alternating directions [Fig. 1(b)]. After comparing the structures written with the opposite sign of the pulse front tilt, the mirror change in the induced modifications is evident [Figs. 1(a) and 1(b)]. This experiment unambiguously demonstrates that the directional dependence of the writing process and the induced modification is determined by the pulse front tilt of the femtosecond laser pulses.

Smooth line structures written in one direction (Fig. 1) correspond to type 2 modifications with the evidence of form birefringence, while the rough structures produced by writing in the opposite direction belong to type 3 modifications, with the evidence of a void formation. At pulse energies below the threshold value of $2 \mu \mathrm{J}$, both lines written in opposite directions reveal type 2 modifications. Moreover, when the pulse energy was increased to $3 \mu \mathrm{J}$ and above, both lines revealed type 3 modifications and the birefringence in the induced structures disappeared. The latter result indicates that the threshold energy for creating type 3 modification inside fused silica depends not only on the pulse energy but also on the writing direction. For example, the line structures, written toward the top in Fig. 1(a), reveal higher threshold energy for the type 3 modifications than the structures written in the opposite direction. This threshold dependence reverses when the sign of the pulse front tilt is changed [Fig. 1(b)]. Furthermore, no directional dependence could be observed when the pulse front tilt was minimized by tuning the pulse compressor.

It should be highlighted that the quill writing effect depends strongly on the focusing depth of the laser irradiation under the sample surface. Changing the focusing depth by only $10 \%$, which is from 60 to 55 or $65 \mu \mathrm{m}$, completely eliminated the quill writing effect and produced type 2 or type 3 structures in both directions.

An alternative experiment was also carried out to provide additional evidence that the pulse front tilt is responsible for the quill writing effect. A laser source, producing pulses of $150 \mathrm{fs}$ duration at $250 \mathrm{kHz}$ repetition rate and $800 \mathrm{~nm}$, was used in this experiment. The laser beam was focused via a $50 \times(\mathrm{NA}=0.55)$ objective at a depth of $\sim 120 \mu \mathrm{m}$ beneath the surface of the sample. The optimum depth for the quill effect in this experiment was different from the previous one due to the difference in laser beam parameters in the two experiments. The first group of four lines with alternating writing directions was imprinted with the pulse energy of $1.4 \mu \mathrm{J}$ and scan speed of $50 \mu \mathrm{m} / \mathrm{s}$ [Fig. 2(a)]. One additional mirror was added in the setup to reverse the direction of the pulse front tilt before writing the next group of lines [Fig. 2(b)]. In this writing, configuration the second group of four lines was imprinted with all other writing parameters identical to the previous experiment. It was observed that the structural modifications in the lines of the second group were mirrored when compared with the lines of the first group. This result provides further evidence that the pulse front tilt is responsible for the directional dependence in the ultrafast laser writing.

We also observed strong white light emission (400-700 $\mathrm{nm}$ ) corresponding to type 3 structural modifications and a weaker emission corresponding to the nanogratings formation, which were reproducible in different writing experiments. Supercontinuum generation can be excluded from the

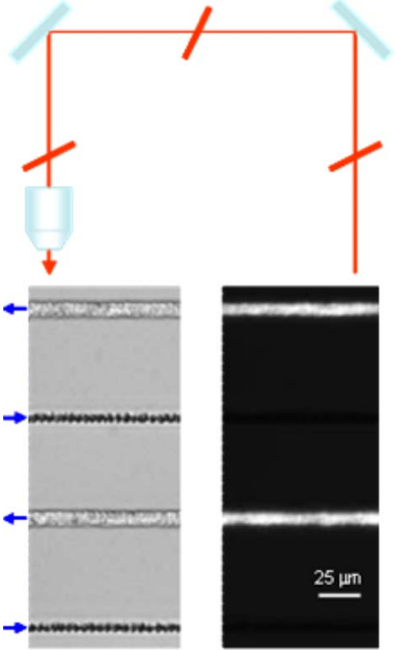

(a)

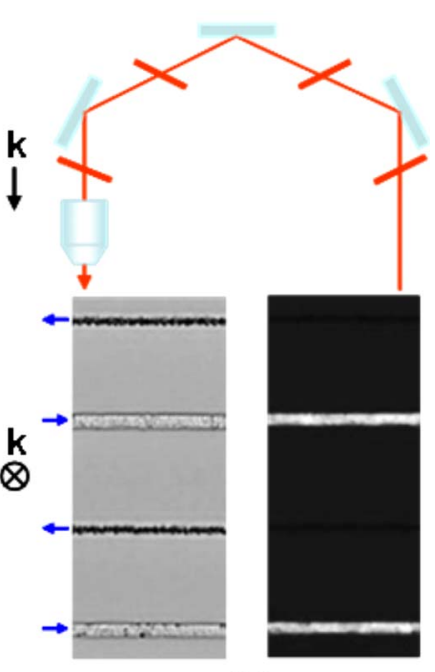

(b)
FIG. 2. (Color online) Microscope images without polarizers (light part) and in crossed polarizers (dark part) of the line structures fabricated with two mirrors (a) and three mirrors (b) in the writing setup at $1.4 \mu \mathrm{J}$ pulse energy. The pulse front is shown by the red line in the setup schematic. $\mathbf{k}$ is the wave vector of the writing laser.

explanation of this emission because the length of light propagation in the focus of high NA objective is too small to produce a significant spectral broadening effect. The observed white emission can be explained by thermal radiation, e.g., bremsstrahlung radiation, emitted by electron plasma which is heated to thousands of degrees at high light intensities of about $10^{14} \mathrm{~W} / \mathrm{cm}^{2} .{ }^{10}$

Another intriguing result was the observation of anisotropic bubble formation, when the pulse energy was increased to the level of $2.4 \mu \mathrm{J}$. Neither birefringence structures (type 2), nor rough features with evidence of void formation (type 3) could be observed in the modified region. Instead, bubbles with diameters varying from 1-3 $\mu \mathrm{m}$ were revealed at the boundary of the irradiated region, which is evident from the cross-sectional image [Fig. 3(c)] and the corresponding top view images of the line structure [Figs. 3(a) and 3(b)]. The color of bubbles visible under white light illumination in the optical microscope [Figs. 3(a) and 3(b)] is attributed to the Fabry-Perot effect. It is well known that voids (type 3) can be produced on the axis of a focused femtosecond laser beam inside transparent material as a result of a microexplosion. ${ }^{4,11}$ However, in our case, the bubbles are formed not in the region of highest intensity (which is in the centre of a Gaussian beam) but are shifted about $5 \mu \mathrm{m}$ toward one side of this center [Figs. 3(b) and $3(\mathrm{c})]$. It should be noted that the movement of bubbles along the direction of writing has been previously demonstrated in fused silica. ${ }^{12}$ Surprisingly, in our experiment, we observed that the bubbles are shifted perpendicular to the writing direction. In addition, we observed bubbles at both sides of the irradiated region when the writing direction was reversed [Fig. 3(a)]. The mechanism of anisotropic bubble formation is a puzzle. Bubbles can be formed on the axis of the focused laser beam as a result of microexplosion and subsequently move from the molten center to the boundary of irradiated region. Alternatively, they can be formed directly at the boundary region of a light affected zone, where tensile stress causes the formation of cavitation bubbles when the rupture strength in molten glass is exceeded. ${ }^{13}$ Clarification of the 


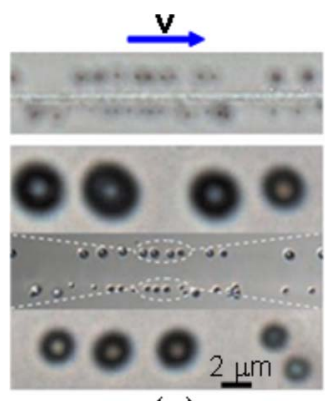

(a)

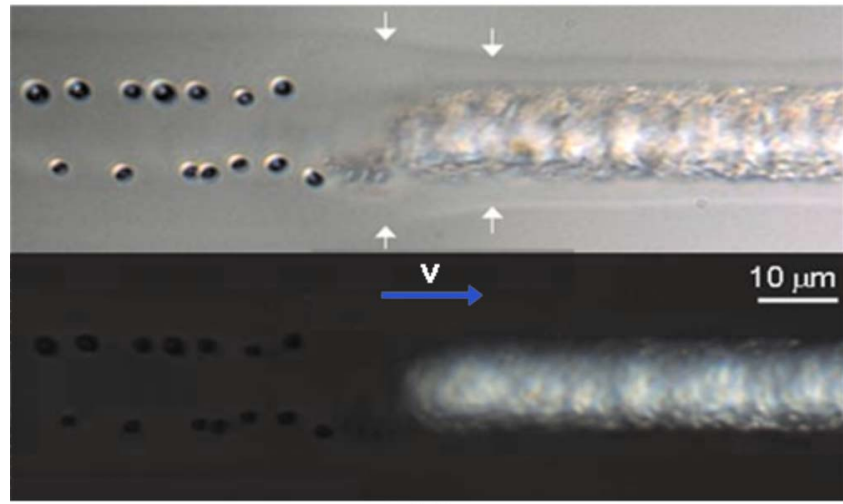

(d)

FIG. 3. (Color online) [(a) and (b)] Bight field images with different magnifications of the line structures fabricated in opposite directions (a) and (b) with $2.4 \mu \mathrm{J}$ pulse energy and scan speed of $50 \mu \mathrm{m} / \mathrm{s}$. (c) Image of a cross section of the line structure shown in (b). (d) Images of the transition region without and with crossed polarizers. $\mathbf{V}$ is the writing velocity, $\mathbf{k}$ is the wave vector of the writing laser, and $\mathbf{E}$ is the electric field of the writing laser.

phenomenon's mechanism needs further investigation. However, we believe that the light pressure at the tilted front of the pulse could be responsible for the asymmetry of bubble formation and its dependence on writing direction.

Another interesting observation was an unusual transition from the regime of bubble formation to self-assembled form birefringence, which occurred during the continuous writing process [Fig. 3(d)]. The phenomenon revealed itself as follows during single line writing. First, bubbles began to appear almost from the start of writing process. At a certain distance, typically about $500 \mu \mathrm{m}$ from the start of writing, the formation of bubbles abruptly terminated. Instead evidence of self-assembled form birefringence appeared and continued until the end of line writing process. The transformation in the type of modification was correlated with the change in intensity of white light emission during the inscription process, which was similar to the emission described above. Strong white light emission during the bubble formation dropped at the modification transition and only weak emission could be observed during the formation of birefringent structures. It should be noted that all writing parameters, including the pulse energy and scanning speed, were kept constant during the encryption process and the phenomenon could be repeated in different areas of the sample. However, there is one parameter which is not constant, in particular the temperature of the whole glass sample, which could increase as a result of light absorption during the writing process. It should be noted that self-assembled form birefringence structures and related nanogratings disap- pear at temperatures above the glass transition temperature. This indicates that local temperature in the irradiated region should drop at modification transition from the glass melting temperature $\left(1715^{\circ} \mathrm{C}\right)$, corresponding to the bubble formation, to the temperature below the glass transition temperature $\left(1175^{\circ} \mathrm{C}\right)$, corresponding to the nanograting formation. This local temperature decrease can be explained by an increase of the heat capacity caused by the heating of the whole glass sample. Indeed, given that $\Delta T=\Delta Q / m c_{p}$, where $\Delta T$ is the temperature difference, $\Delta Q$ is the heat energy, and $m$ is the mass of the substance, $c_{p}$ is the heat capacity, the temperature drops when heat capacity increases. The nanograting starts to form once the local temperature has dropped below the glass transition temperature. The energy is consumed by a nanograting formation process, which locks temperature below the glass transition temperature and explains why formation of nanogratings does not stop until the end of writing process.

In conclusion, a change in structural modification was demonstrated in silica glass sample by controlling the direction of pulse front tilt, achieving a calligraphic style of laser writing which is similar in appearance to that inked with the bygone quill pen. Moreover, anisotropic bubble formation, which could be controlled by writing direction, was observed at high pulse energies. The phenomenon of modification transition in a continuous encryption process was observed. Our experiments provide the comprehensive evidence that the light beam with centrosymmetric intensity distribution can produce noncentrosymmetric material modifications. We anticipate that the possibility of achieving control of lightmatter interactions by adjusting the tilt of ultrashort pulse front could lead to advances in material processing, optical manipulation and data storage.

The work was supported by the Physical Sciences Research Council (EPSRC) and New Energy and Industrial Technology Development Organization (NEDO).

${ }^{1}$ R. Birngruber, C. A. Puliafito, A. Gawande, W. Z. Lin, R. W. Schoenlein, and J. G. Fujimoto, IEEE J. Quantum Electron. 23, 1836 (1987).

${ }^{2}$ K. M. Davis, K. Miura, N. Sugimoto, and K. Hirao, Opt. Lett. 21, 1729 (1996).

${ }^{3}$ E. N. Glezer, M. Milosavljevic, L. Huang, R. J. Finlay, T. H. Her, J. P. Callan, and E. Mazur, Opt. Lett. 21, 2023 (1996).

${ }^{4}$ B. Poumellec, L. Sudrie, M. Franco, B. Prade, and A. Mysyrowicz, Opt. Express 11, 1070 (2003).

${ }^{5}$ W. J. Yang, P. G. Kazansky, and Y. P. Svirko, Nat. Photonics 2, 99 (2008).

${ }^{6}$ Y. Shimotsuma, P. G. Kazansky, J. R. Qiu, and K. Hirao, Phys. Rev. Lett. 91, 247405 (2003).

${ }^{7}$ W. J. Yang, E. Bricchi, P. G. Kazansky, J. Bovatsek, and A. Y. Arai, Opt. Express 14, 10117 (2006).

${ }^{8}$ P. G. Kazansky, W. J. Yang, E. Bricchi, J. Bovatsek, A. Arai, Y. Shimotsuma, K. Miura, and K. Hirao, Appl. Phys. Lett. 90, 151120 (2007).

${ }^{9}$ S. Akturk, M. Kimmel, P. O'Shea, and R. Trebino, Opt. Express 11, 491 (2003).

${ }^{10}$ R. Graf, A. Fernandez, M. Dubov, H. J. Brueckner, B. N. Chichkov, and A. Apolonski, Appl. Phys. B: Lasers Opt. 87, 21 (2007).

${ }^{11}$ S. Juodkazis, K. Nishimura, S. Tanaka, H. Misawa, E. G. Gamaly, B. Luther-Davies, L. Hallo, P. Nicolai, and V. T. Tikhonchuk, Phys. Rev. Lett. 96, 166101 (2006).

${ }^{12}$ W. Watanabe and K. Itoh, Opt. Express 10, 603 (2002).

${ }^{13}$ A. Vogel, J. Noack, G. Huttman, and G. Paltauf, Appl. Phys. B: Lasers Opt. 81, 1015 (2005). 